\title{
Secondary Phosphine Oxide-Activated Nickel Catalysts for Site-Selective Alkene Isomerization and Remote Hydrophosphination
}

\author{
Lin Huang ${ }^{1}$, En Qi Lim ${ }^{1} \&$ Ming Joo Koh ${ }^{1 *}$ \\ ${ }^{1}$ Department of Chemistry, National University of Singapore, 4 Science Drive 2, Republic of Singapore, 117544.
}

\begin{abstract}
Catalytic systems that are readily modifiable to achieve olefin migration or remote functionalization are highly sought-after in chemical synthesis. Here, we show that the combination of a commercially available nickel(II) pre-catalyst and a secondary phosphine oxide ligand enables site- and stereoselective alkene transposition for up to nine double-bond migrations within terminal and internal olefins under mild reductive conditions. Substrates bearing diverse functionalities including Brønsted acidic and reducible carbonyl groups are tolerated. Mechanistic and spectroscopic studies revealed the in situ generation of a catalytically active nickel-hydride species triggered by oxidative addition of the phosphine oxide. The reaction is amenable to regioconvergent isomerization as well as $\beta$-selective remote hydrophosphination when stoichiometric secondary phosphine oxide/base were employed.
\end{abstract}

The transposition of carbon-carbon double bonds ${ }^{1,2}$ is arguably one of the most convenient approaches to convert an easily available olefin to another unsaturated molecule of interest, and has vast applications in various chemical industries 3,4 . Combining olefin isomerization with another functionalization reaction enables functional groups to be precisely incorporated at positions remote from the initial reaction site ${ }^{1,5-8}$. Such transformations offer new disconnection strategies in organic synthesis by allowing different substrates (e.g. regioisomeric alkene mixtures) ${ }^{1,5-8}$ to be employed, and unlock new reactivity modes through formal $\left(\mathrm{sp}^{3}\right) \mathrm{C}-\mathrm{H}$ functionalization at typically less-activated sites $^{9}$. Methodologies using catalyst systems derived from noble metals (e.g., Pd, Ir) ${ }^{10-16}$ and base metals (e.g., Ni, Fe) ${ }^{17-24}$ have been conceived for alkene isomerization over the years. Nonprecious nickel catalysis represents an attractive platform for reaction development. Until recently, however, strategies that involve nickel-based complexes typically employ harsh additives (e.g. strong acids ${ }^{25-27}$ and hydrides ${ }^{28-30}$ ) or high reaction temperatures ${ }^{31}$ to generate the active $\mathrm{Ni}-\mathrm{H}$ species from $\mathrm{Ni}(0)$ precursors, consequently compromising functional group compatibility (Fig. 1a). A recent remarkable method ${ }^{20}$ utilized a somewhat air-sensitive dimeric $\mathrm{Ni}(\mathrm{I})$ complex $^{32}$ to mediate efficient $\mathrm{C}=\mathrm{C}$ bond migrations over 
multiple positions, although isomerizations of internal olefins and regioisomeric mixtures are difficult ${ }^{18}$.

Our recent work in catalytic olefin migrations ${ }^{18}$ and tandem processes ${ }^{9}$ led us to wonder if we can develop a new catalytic isomerization manifold using commercially available and inexpensive starting materials/reagents. Specifically, we sought to devise a versatile system that can be selectively tuned to generate either olefin isomerization or remote hydrofunctionalization ${ }^{1,5-}$ $8,33,34$ products. To this end, we questioned whether an appropriate mild hydride source in conjunction with a base metal complex can be identified to generate the requisite metal-hydride ${ }^{1}$ species to promote chain-walking, and whether conditions can be readily modified to trigger further transformations following isomerization.
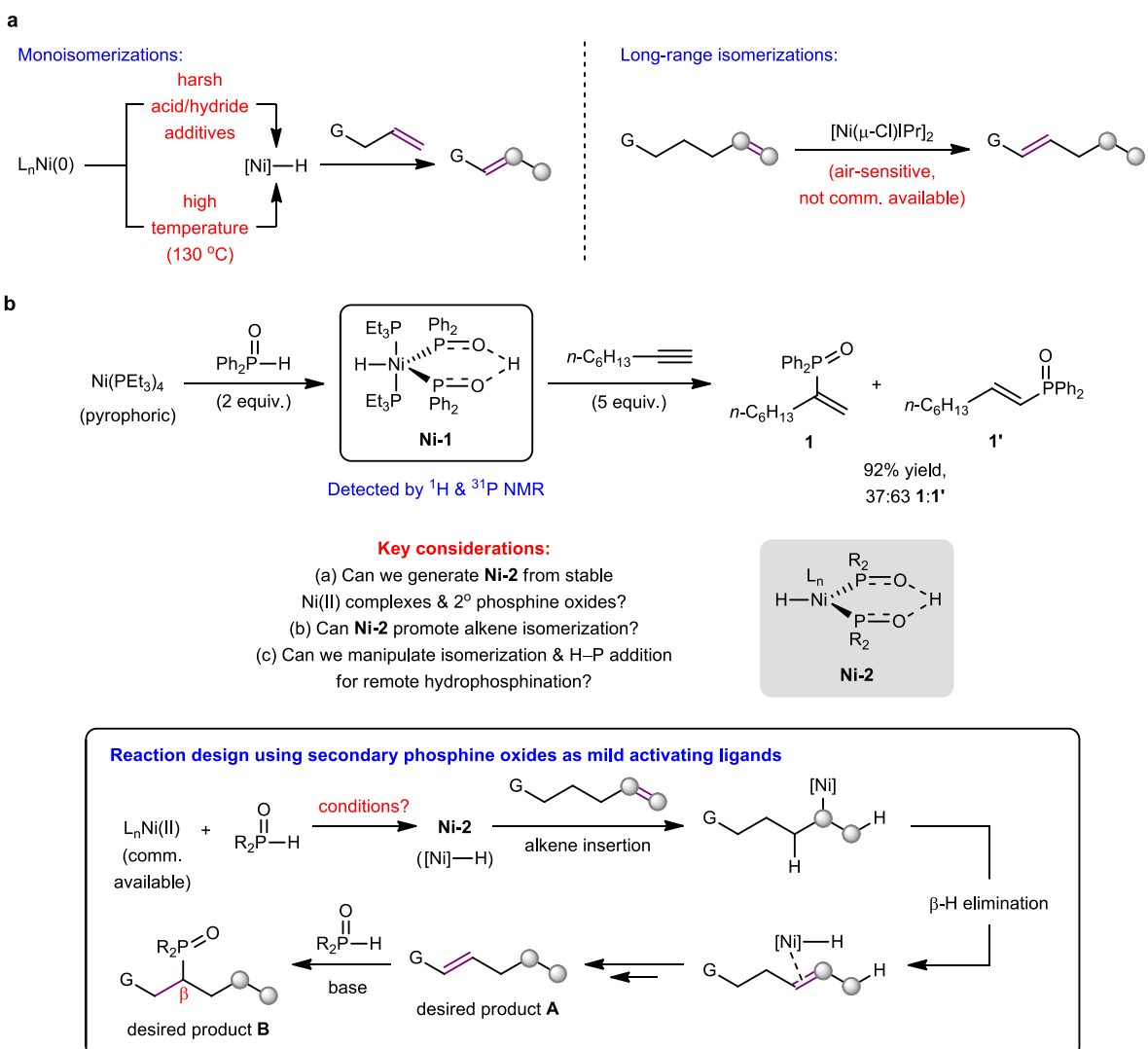

c

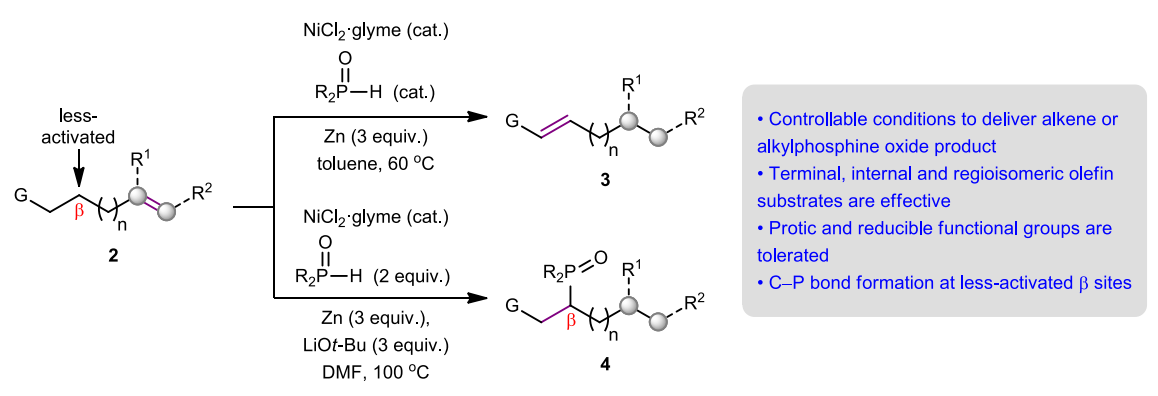


Fig. 1. The significance of designing an alkene isomerization/remote functionalization regime using nickel catalysis and secondary phosphine oxides as mild activating ligands. a, Representative Ni-catalyzed strategies for alkene isomerization and their associated limitations. $\mathbf{b}$, Secondary phosphine oxides are previously reported to promote the formation of nickel-hydride species for alkyne hydrophosphination. However, it is unknown whether such a species can be generated from stable $\mathrm{Ni}$ (II) precursors and phosphine oxides under operationally mild conditions. If successful, the nickel-hydride species should be capable of mediating olefin chain-walking through sequential insertions/ $\beta-\mathrm{H}$ eliminations. c, Development of a reductive $\mathrm{Ni}$-catalyzed manifold that offers tunable access to valuable regiodefined alkene isomerization or remote hydrophosphination products.

In light of these deliberations, we turned to pentavalent secondary phosphine oxides ${ }^{35-39}$, which are known to tautomerize to trivalent phosphinous acids that serve as phosphane-type ligands when exposed to a transition metal-based complex ${ }^{40-42}$. Han and co-workers reported that a phosphine-nickel(0) complex could mediate regioselective $\mathrm{H}-\mathrm{P}$ additions to alkynes using phosphine oxides as reagents ${ }^{43}$. Experimental evidence pointed to the intermediacy of a hydrogen bonding-stabilized five-coordinate nickel-hydride ${ }^{43}$ complex $\mathbf{N i - 1}$ that drives hydronickelation followed by $\mathrm{C}\left(\mathrm{sp}^{2}\right)-\mathrm{P}$ bond-forming reductive elimination (Fig. 1b). Intrigued by these seminal observations, we envisioned that a catalytic combination of an organonickel(II) complex and a secondary phosphine oxide may be leveraged to furnish a putative nickel-hydride Ni-2 analogous to $\mathbf{N i - 1}$, in which the non-innocent ${ }^{40}$ phosphine oxide plays a role as both a Lewis basic ligand and hydride donor. Although unprecedented, we surmised that such complexes should be capable of promoting olefin isomerization through consecutive $\mathrm{Ni}-\mathrm{H}$ addition and $\beta-\mathrm{H}$ elimination ${ }^{1,5-8}$ under appropriate parameters.

Moreover, when stoichiometric phosphine oxide is used in the presence of a base ${ }^{44-47}$, remote hydrophosphination may be potentially implemented. However, manipulating alkene isomerization to precede hydrophosphination can be challenging, and a slow chain-walking process will inevitably result in premature phosphinyl additions and hence undesirable regioisomeric product mixtures ${ }^{45,48}$. If successfully obtained, the $\alpha$-branched alkylphosphine oxides not only function as stable precursors of the oxidation-labile phosphines ${ }^{49-51}$, but are also useful as Lewis bases ${ }^{52-54}$ and ligands in organometallic chemistry ${ }^{35-42}$. To the best of our knowledge, there is no precedence of remote alkene hydrophosphination in which the phosphinyl unit is installed at less-reactive sites vicinal $(\beta)$ to a pendant entity ${ }^{9}$. This will open up unexplored avenues towards the preparation of novel organophosphorus compounds for diverse applications such as drug synthesis ${ }^{55,56}$ and ligand design ${ }^{57,58}$. Herein, we report our findings in Ni-mediated olefin migration and remote hydrophosphination under reductive conditions (Fig. 1c).

\section{Results}


Reaction optimization. We commended our studies by examining conditions (see Supplementary Information (SI) Section 2 for details) for the complete isomerization of terminal olefin $\mathbf{2 a}$ to $\mathbf{3 a}$ (Table 1). After extensive experimentation, best results could be achieved using commercially available $\mathrm{NiCl}_{2} \cdot$ glyme (10 mol \%) and diphenylphosphine oxide $\mathbf{L 1}$ (20 mol \%) with excess $\mathrm{Zn}$ as reducing agent and toluene as solvent, furnishing 3a in 79\% GC yield (78\% isolated yield) exclusively as the $E$ isomer at $60^{\circ} \mathrm{C}$ (entry 1 ). Substituting the ligand on the $\mathrm{Ni}(\mathrm{II})$ chloride complex had a non-negligible impact on isomerization efficiency, with more electron-donating phosphines giving greater amounts of undesired alkene regioisomers 3a' and hydrogenated 3a' (entry 2). Other $\mathrm{Ni}(\mathrm{II})$ salts were significantly less effective in promoting olefin migration (entries $3-4)$. With reference to Fig. $1 b$, we speculated that a ligated $\mathrm{Ni}(0)$ species was generated in situ by reduction of a $\mathrm{Ni}(\mathrm{II})$ complex ${ }^{59,60}$ prior to activation by L1. However, isomerization was inefficient with $\mathrm{Ni}(\operatorname{cod})_{2}$ as pre-catalyst, and adding catalytic (or stoichiometric) quantities of DME as a coordinating ligand did not improve results substantially (entry 5).

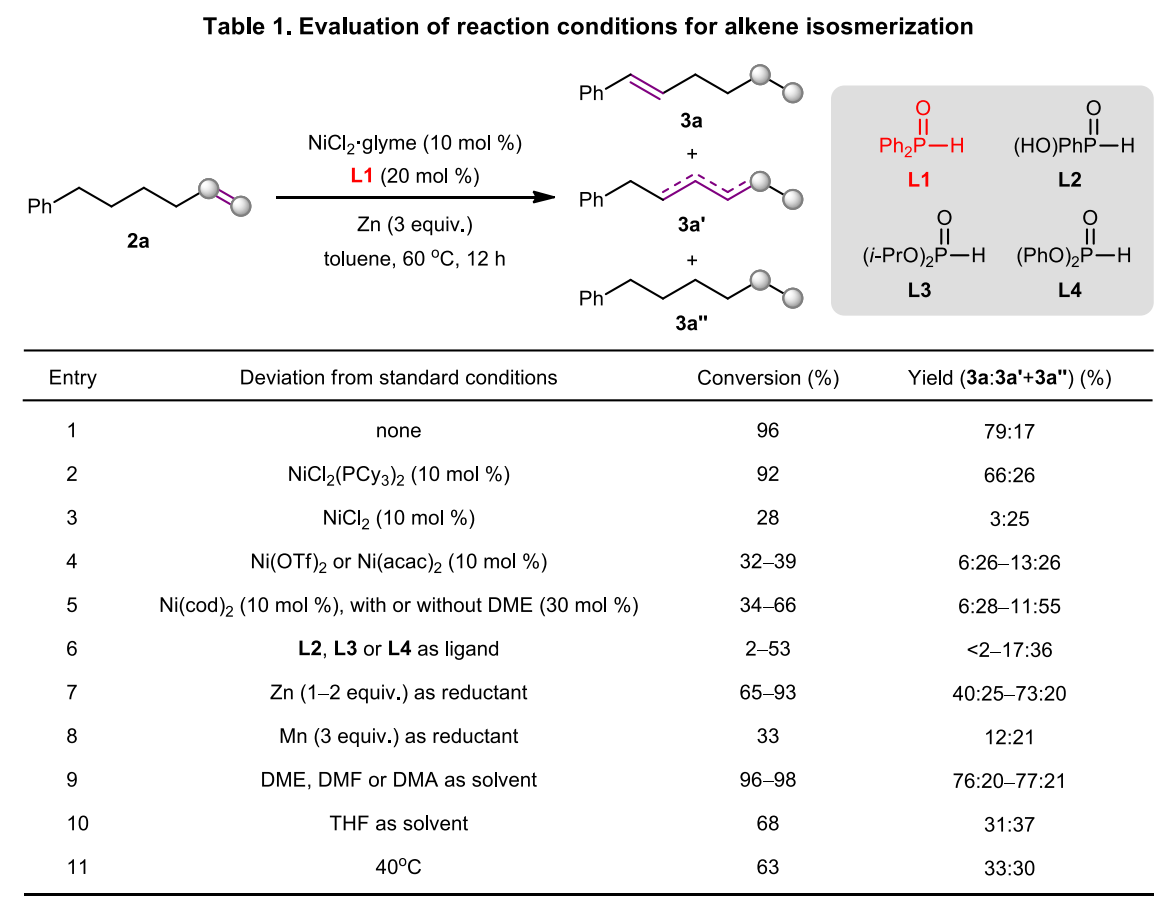

Reactions were carried out on $0.1 \mathrm{mmol}$ scale. Conversions and yields were determined by GC analysis with $\mathrm{n}$-decane as an internal standard. 3a was obtained in $>98: 2 E: Z$ ratio in all cases.

Other commercially available secondary phosphine oxides L2-L4 were incompetent in promoting olefin transposition (entry 6), while reducing the $\mathrm{Zn}$ loading or changing the reductant had deleterious effects (entries 7-8). The alkene transposition could be conducted in polar solvents such as DME, DMF and DMA with similar chain-walking efficiency (entry 9), but THF was 
visibly less effective (entry 10). Reaction temperature was crucial since poorer conversion to an unsatisfactory mixture was detected at $40^{\circ} \mathrm{C}$ (entry 11 ).

Site- and stereoselective alkene isomerization. We proceeded to evaluate a range of functionalized terminal and internal alkene substrates under the established olefin migration conditions (Fig. 2). Monoisomerizations could be accomplished with monosubstituted olefins to deliver a diverse assortment of di- and trisubstituted products in 70-96\% yield and >98:2 E:Z ratios. These include styrenyl products bearing electron-deficient (3b-3c) and electron-rich $(\mathbf{3 d}-\mathbf{3 g})$ arenes as well as those containing reactive functionalities such as a reducible ketone (3b), nitrile (3c) and Brønsted acidic phenol (3d). Boryl and silyl units were also amenable migration terminus, enabling access to synthetically valuable 1,2-disubstituted alkenylboronates $^{61}(\mathbf{3 h})$ and alkenylsilanes ${ }^{62}(\mathbf{3 i}-\mathbf{3} \mathbf{j})$. Starting from monosubstituted or exocyclic 1,1-disubstituted substrates, transformations leading to sterically demanding trisubstituted cyclic $(\mathbf{3 k}-\mathbf{3 l})$ or acyclic $(\mathbf{3 m}) \mathrm{C}=\mathrm{C}$ bonds proceeded without a diminution in reaction efficiency.

Longer-distance migrations could be similarly induced, furnishing the fully isomerized internal alkenes $\mathbf{3 n}-\mathbf{3 z}$ in $54-90 \%$ yield and $>98 \% E$ selectivity. The reaction could be performed on gram scale with comparable efficiency $(3 n)$, highlighting the robustness of the catalytic regime. Synthesis of $\mathrm{N}$-alkenyl indole $\mathbf{3 t}$ shows that isomerization may be terminated by a nitrogen group. Notably, E-3z was isolated cleanly in $54 \%$ yield from a chain-walking process featuring nine double-bond shifts, comparing favorably with past reports ${ }^{18,20} .1,2$-Disubstituted internal olefins also underwent migration to give the desired products (3aa-3ab), which is complementary in scope to a previous $\mathrm{Ni}$-catalyzed report ${ }^{20}$. However, tri- and tetrasubstituted $\mathrm{C}=\mathrm{C}$ bonds were unreactive. The opportunity then arises to test the limits of our protocol in regioconvergent isomerization where a complex mixture of four regioisomeric substrates $2 \mathbf{2 a}, \mathbf{5}-\mathbf{7}$ (each containing $E$ and $Z$ isomers) was subjected to the standard conditions. In the event, $E-3 x$ was secured in $81 \%$ yield as the sole product, providing an attractive streamlined approach to convert mixtures of low-cost olefin feedstock to value-added isomerically pure products. 

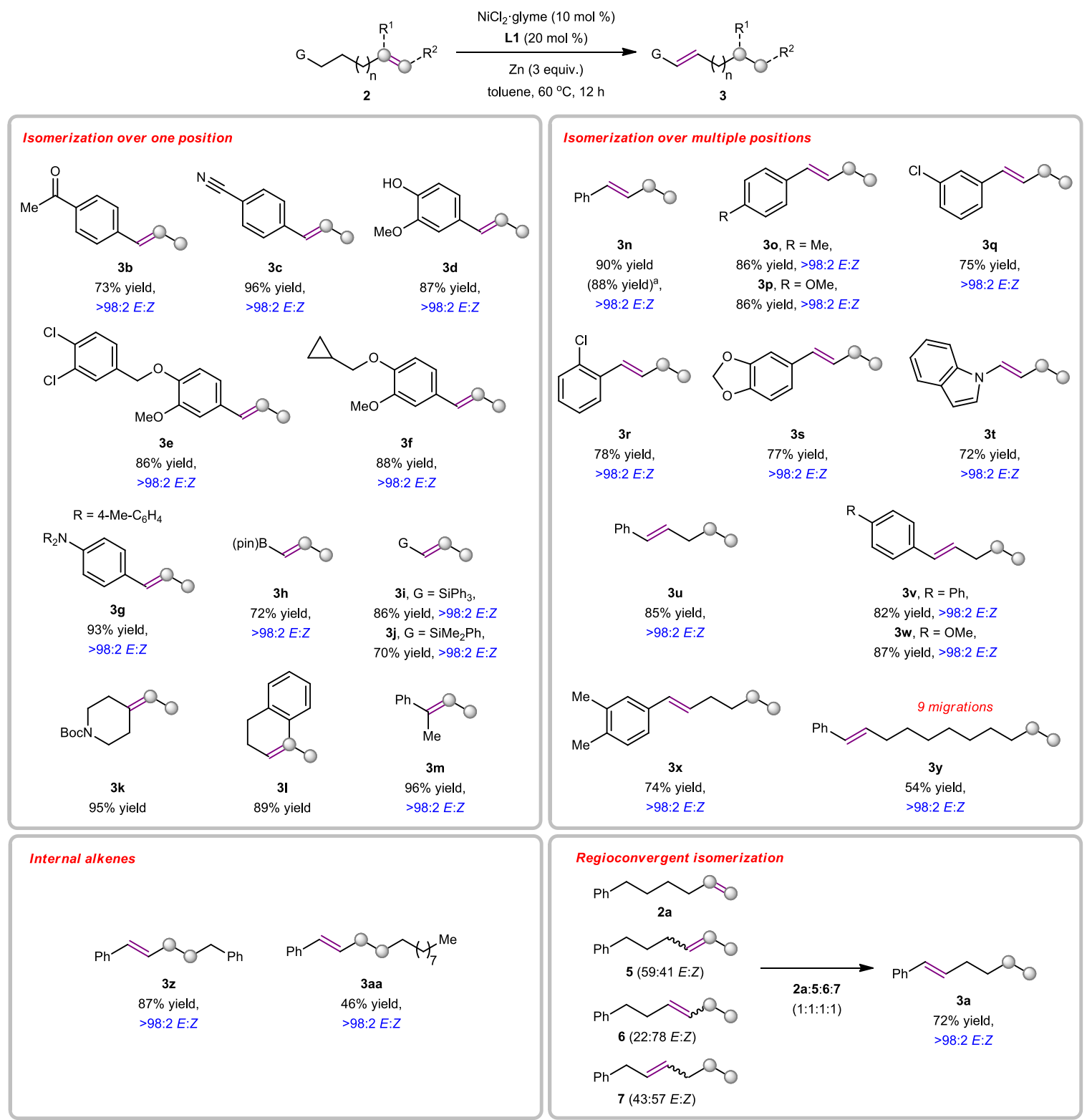

Fig. 2. The scope of Ni-catalyzed alkene isomerization. Reactions were carried out on $0.2 \mathrm{mmol}$ scale. E:Z ratios were determined by ${ }^{1} \mathrm{H}$ NMR analysis of the isolated and purified products. Yields are for isolated and purified products. ${ }^{\text {TThe reaction was }}$ conducted on $10 \mathrm{mmol}$ scale.

$\beta$-Selective remote hydrophosphination. We then investigated the feasibility of implementing remote alkene hydrophosphination (Fig. 3). Control experiments indicated that addition of stoichiometric L1 did not promote hydrophosphination in the absence of base (only chainwalking observed, which suggests that alkene isomerization precedes hydrophosphination). Although base-mediated 1,2-hydrophosphination of activated alkenes ${ }^{45-47}$ has been documented, it remains to be determined if our Ni-mediated chain-walking system is compatible with exogenous bases, and whether the two processes of olefin migration and H-P addition can 
be controlled to selectively incorporate phosphinyl groups. After surveying various parameters, the reaction of $\mathbf{2 a}$ afforded alkylphosphine oxide 4 a bearing a $\beta$-phosphionyl unit in $71 \%$ isolated yield as a single regioisomer, using the $\mathrm{NiCl}_{2} \cdot g$ lyme/L1/Zn system in DMF in the presence of excess LiOt-Bu (optimal base) at $100{ }^{\circ} \mathrm{C}$. Decreasing the base loading proved detrimental to efficiency (primarily olefin isomerization detected with $15 \%$ yield of 4 a using 1 equiv. of base), and less polar solvents (e.g. toluene) were ineffective (see SI Table S3 for details).

Regardless of the chain length, reliable P-installation at the $\beta$-position ( $>98 \%$ regioselectivity across the board) was obtained for a wide array of alkenes containing arenes (4b-4f, $\mathbf{4 h}-\mathbf{4 k}, \mathbf{4 n}$ ), heteroarenes $(\mathbf{4 g})$ and amides $(\mathbf{4 l}-\mathbf{4 m})$, delivering adducts in up to $89 \%$ yield. Secondary amides with an acidic $\mathrm{N}-\mathrm{H}$ unit (4I) are tolerated, and 1,1-disubstituted $\mathrm{C}=\mathrm{C}$ bonds (4h) also participated in remote hydrophosphination. The exclusive generation of $\mathbf{4 n}$ as the $\beta$-isomer from the corresponding long-chain alkene underscores the remarkable fidelity of the reaction. Dialkylphosphine oxide L5 was also competent in promoting regiocontrolled chainwalking/phosphinyl addition, giving rise to the corresponding product $\mathbf{4 0}$ in $81 \%$ yield.
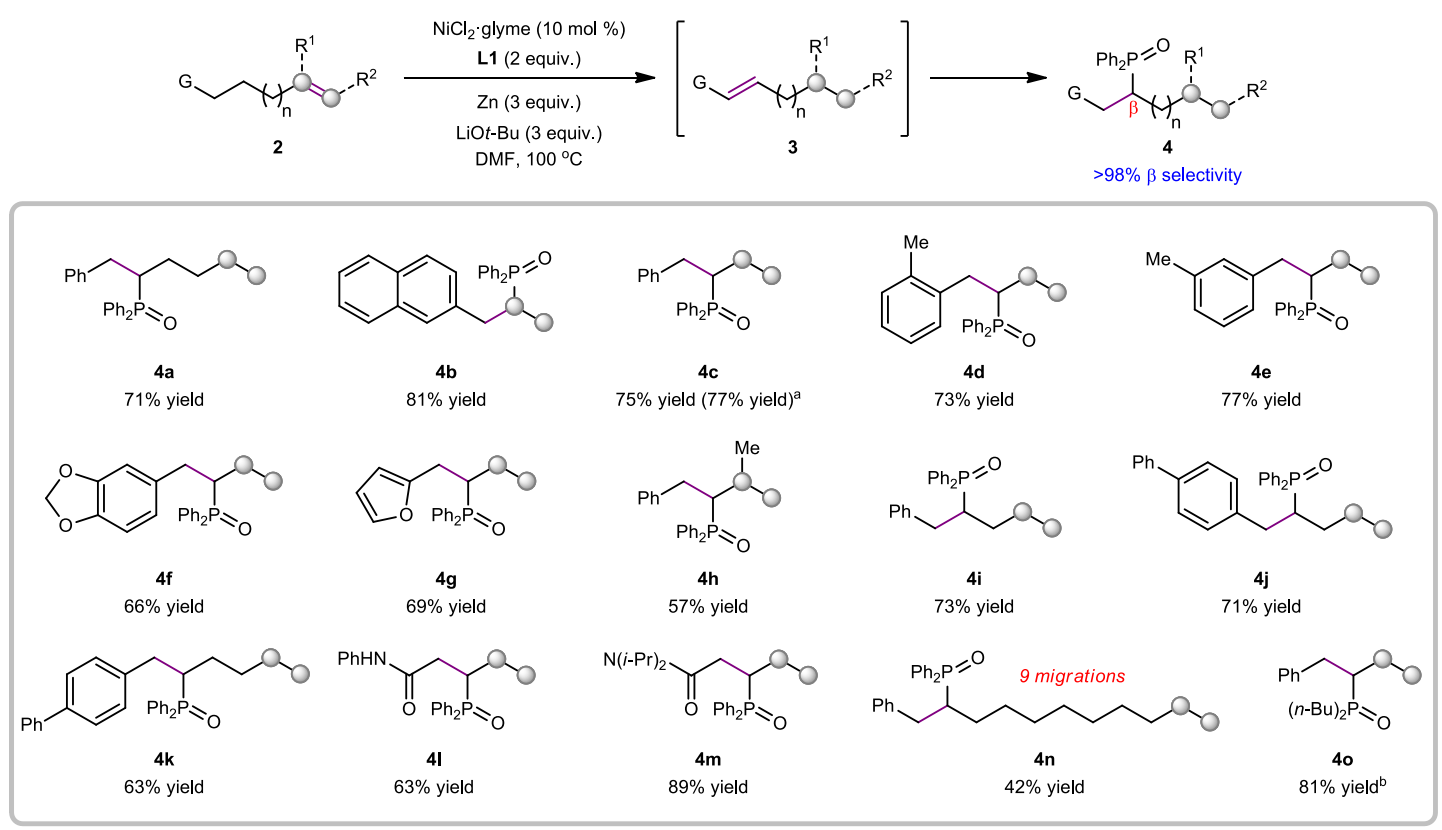

Fig. 3. $\beta$-Selective remote hydrophosphination. Reactions were carried out on $0.2 \mathrm{mmol}$ scale. Regioisomeric ratios were determined by ${ }^{1} \mathrm{H}$ NMR analysis of the isolated and purified products. Yields are for isolated and purified products. ${ }^{\text {aThe reaction }}$

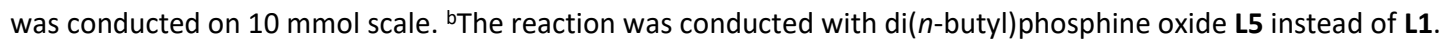

Mechanistic investigations. ${ }^{1} \mathrm{H}$ and ${ }^{31} \mathrm{P}$ NMR studies were conducted to obtain information on the in situ-generated organonickel species (Fig. 4a). Specifically, we sought to acquire evidence 
for the formation of a catalytically active nickel-hydride complex responsible for olefin transposition.

a

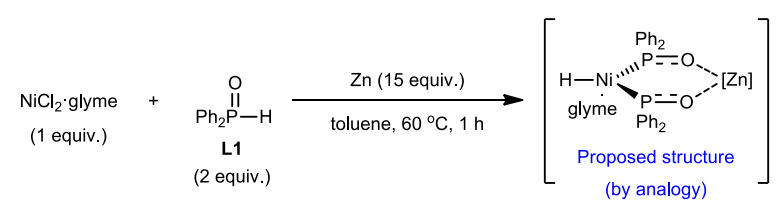

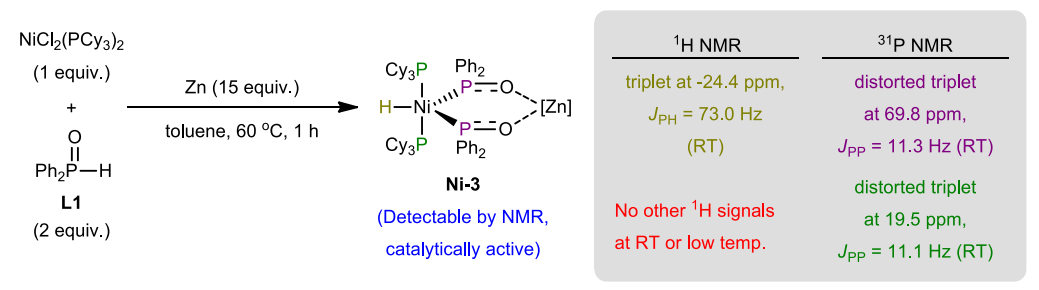

b

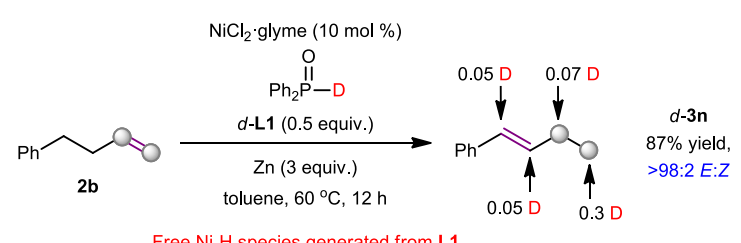

c
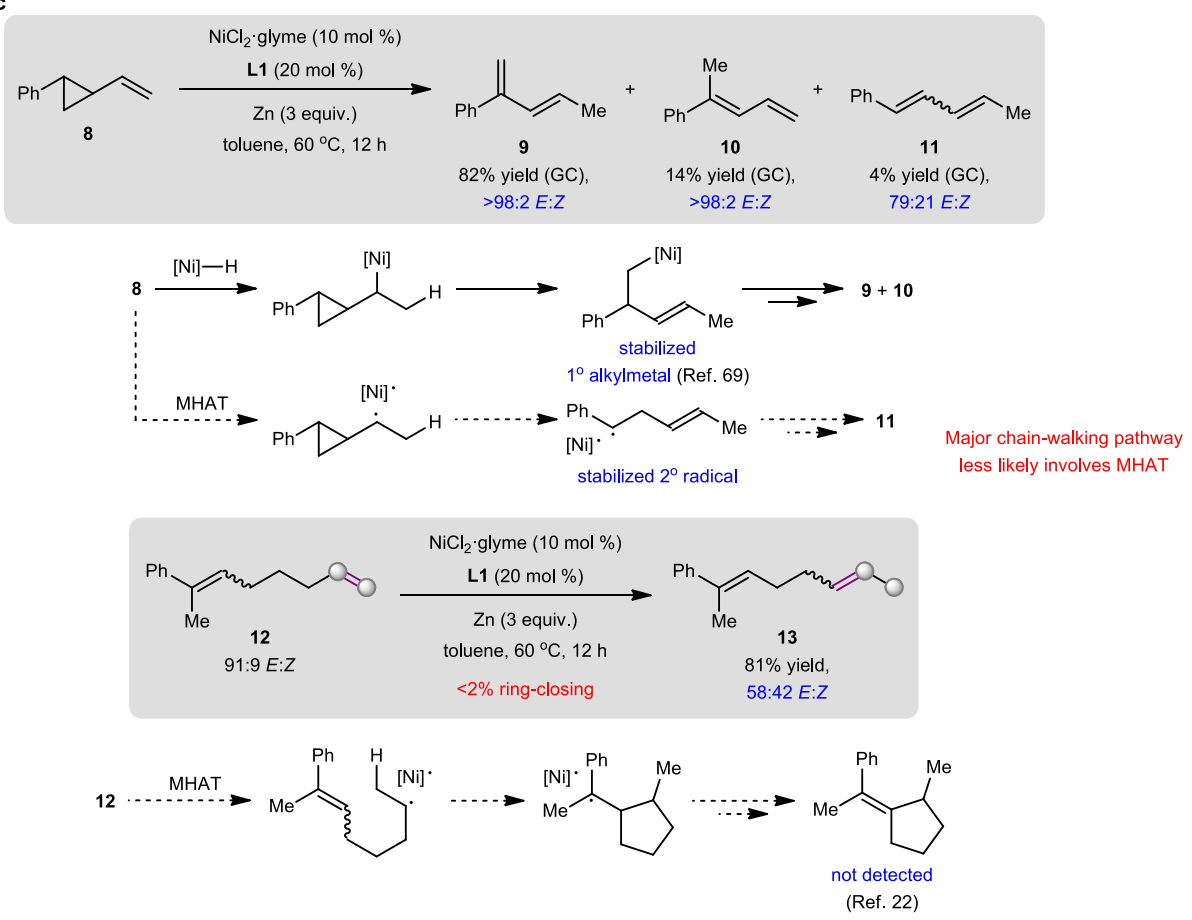

Fig. 4. Mechanistic studies. a, NMR studies to probe the nature of the in situ-generated nickel-hydride species. $\mathbf{b}$, Deuterium labeling experiment. c, Radical clock experiments. Reactions were carried out on $0.1 \mathrm{mmol}$ scale. E:Z ratios were determined by GC analysis with $n$-decane as an internal standard. Yields are for isolated and purified products, unless otherwise stated.

However, initial attempts to decipher through spectroscopic analysis by treating a 1:2 mixture of $\mathrm{NiCl}_{2} \cdot$ glyme and $\mathbf{L} \mathbf{1}$ in toluene with excess $\mathrm{Zn}$ at various temperatures were unsuccessful, which 
might be attributed to the instability and short lifetime $41,43,63-65$ of the putative nickel species. In order to derive meaningful data, we selected $\mathrm{NiCl}_{2}\left(\mathrm{PC}_{3}\right)_{2}$ with strongly coordinating tricyclohexylphosphine ligands (vs. glyme) as the nickel precursor, which was also reasonably efficient in promoting chain-walking (Table 1 entry 2). In contrast to Han's disclosure ${ }^{43}$ in which a phosphine-nickel(0) complex was employed, our study utilized a $\mathrm{Ni}(I I)$ pre-catalyst which we hypothesized, served as a stable precursor of an organonickel(0) species by reduction with $\mathrm{Zn}^{59}$. Gratifyingly, a sufficiently stable nickel-hydride intermediate Ni-3 that is postulated to adopt a symmetric five-coordinate geometry similar to $\mathbf{N i - 1}{ }^{43}$ (cf. Fig. 1b) was detected. Ni-3 was surmised to derive from oxidative addition of an equivalent of $\mathbf{L} \mathbf{1}$ and coordination by another equivalent of its tautomer ${ }^{35-43}$.

At ambient temperature, the NMR signals arising from L1 disappeared and were subsequently replaced with new signals: a triplet at $-24.4 \mathrm{ppm}\left(\mathrm{J}_{\mathrm{PH}}=73.0 \mathrm{~Hz}\right)$ in the ${ }^{1} \mathrm{H}$ NMR spectrum $(\mathrm{Ni}-\mathrm{H})$ and two distorted triplets $\left(J_{\mathrm{PP}} \sim 11.2 \mathrm{~Hz}\right.$; integration ratio 1:1) at $69.8(\mathrm{P}=0)$ and $\left.19.5 \mathrm{ppm}(\mathrm{PCу})_{3}\right)$ in the ${ }^{31} \mathrm{P}$ NMR spectrum were recorded; these signals were assigned by comparison with reported spectroscopic data ${ }^{43}$. Unexpectedly, no other ${ }^{1} \mathrm{H}$ signals were detected at ambient or cryogenic temperatures, suggesting the apparent absence of a proton bridge that exists in Ni-1 or Ni-2. Instead, the acidic proton could be replaced by a $\mathrm{Zn}(\mathrm{II})$ species $^{41,66-68}$ generated during the course of $\mathrm{NiCl}_{2}\left(\mathrm{PCy}_{3}\right)_{2}$ reduction. Control experiments showed that mixing $d$ - $\mathbf{L} 1$ with $\mathrm{ZnCl}_{2}$ led to disappearance of the P-D signal in the ${ }^{2} \mathrm{H}$ NMR spectrum (see SI Section 7b for details). Unfortunately, crystals of Ni-3 that are suitable for X-ray crystallographic analysis were not obtained. Nevertheless, the mixture that presumably contains Ni-3 was shown to be catalytically active by subjecting it to a terminal olefin, which furnished the fully isomerized product. By analogy, we presumed that a similar monomeric L1-activated nickel-hydride was also generated from $\mathrm{NiCl}_{2}$.glyme through preliminary mass spectrometry analysis, although the formation of oligomeric species could not be completely excluded. Further investigations are underway to elucidate more insights on these in situ-generated organonickel species.

Isomerization of $\mathbf{2} \mathbf{b}$ with $d$ - $\mathbf{L} \mathbf{1}$ resulted in deuterium incorporation and scrambling across the entire hydrocarbon skeleton, providing additional support that the hydride source required to form the active nickel-hydride catalyst originates from the phosphine oxide (Fig. 4b). Radical clock studies were performed with 8 and 12 (Fig. 4c). In the event, 9 (82\% yield) and 10 (14\% yield) were predominantly obtained from $\mathbf{8}^{69}$, whereas 12 was converted to the monoisomerization product 13 in $81 \%$ yield and 58:42 E:Z ratio (<2\% ring-closing detected). ${ }^{22}$ These observations 
intimate that olefin migration involving metal-hydride atom transfer (MHAT) ${ }^{33,34}$ via long-lived radical intermediates is less likely. To summarize, alkene transposition probably proceeds through sequential $\mathrm{Ni}-\mathrm{H}$ olefin insertions/ $\beta$-H eliminations ${ }^{1,5-8}$ instead of 1,3 -hydride shift ${ }^{5-8,70}$ or MHAT.

Control experiments further showed that hydrophosphination likely proceeded by nucleophilic addition of a deprotonated hydroxyphosphine tautomer across the $\mathrm{C}=\mathrm{C}$ bond to give an electronically stabilized carbanion ${ }^{44}$, which could either abstract a proton from hydroxyphosphine or undergo adventitious protonolysis with trace $t$ - $\mathrm{BuOH}$ formed in solution. The reaction of a 1,2-disubstituted aryl alkene with $d$-L1 in the presence of LiOt-Bu afforded the expected hydro(deutero)phosphination adduct (see SI Section 7c for details). Under the basic conditions, H/D exchange was detected at the acidic $\mathrm{C}_{\alpha}-\mathrm{H}$ site adjacent to the electronwithdrawing $\mathrm{P}=\mathrm{O}$ group (i.e. $t-\mathrm{BuOH}$ is inadvertently generated ${ }^{44,49}$ ). Thus, the exceptional $\beta$ selectivity observed in remote hydrophosphination could be rationalized by the lack of undesired phosphinyl additions during the course of chain-walking, since generation of destabilized alkylsubstituted carbanion intermediates is likely unfavorable.

\section{Discussion}

In conclusion, an operationally simple Ni-catalyzed reductive protocol for regio- and stereoselective alkene isomerization was devised. Beyond serving as an anciliary ligand, the secondary phosphine oxide underwent reaction to form the requisite nickel-hydride that promotes non-radical $\mathrm{C}=\mathrm{C}$ bond shifts under mild conditions. The versatility of the present regime allows for regiocontrolled chain-walking/hydrophosphination to occur in one operation, furnishing prized organophosphorus compounds that are difficult to obtain by alternative means. The present work offers a new blueprint for olefin chain-walking that will likely facilitate efforts to tackle other unresolved challenges in site-selective remote alkene functionalization.

\section{References}

1. Larionov, E., Li, H. \& Mazet, C. Well-defined transition metal hydrides in catalytic isomerizations. Chem. Commun. 50, 9816-9826 (2014).

2. Hassam, M., Taher, A., Arnott, G. E., Green, I. R. \& van Otterlo, W. A. L. Isomerization of allylbenzenes. Chem. Rev. 115, 5462-5569 (2015). 
3. Larsen, C. R. \& Grotjahn, D. B. The value and application of transition metal catalyzed alkene isomerization in industry. In Applied Homogeneous Catalysis with Organometallic Compounds: A Comprehensive Handbook in Four Vol.s, Cornils, B., Herrmann, W. A., Beller, M. \& Paciello, R., Eds. Wiley, (2017), pp 1365-1378.

4. Donohoe, T. J., O’Riordan, T. J. C. \& Rosa, C. P. Ruthenium-catalyzed isomerization of terminal olefins: Applications to synthesis. Angew. Chem. Int. Ed. 48, 1014-1017 (2009).

5. Fiorito, D., Scaringi, S. \& Mazet, C. Transition metal-catalyzed alkene isomerization as an enabling technology in tandem, sequential and domino processes. Chem. Soc. Rev. 50, 1391-1406 (2021).

6. Janssen-Müller, D., Sahoo, B., Sun, S.-Z. \& Martin, R. Tackling remote sp3 C-H functionalization via Ni-catalyzed "chain-walking" reactions. Isr. J. Chem. 60, 195-206 (2020).

7. Sommer, H., Juliá-Hernández, F., Martin, R. \& Marek, I. ACS Cent. Sci. 4, 153-165 (2018).

8. Vasseur, A., Bruffaerts, J. \& Marek, I. Remote functionalization through alkene isomerization. Nat. Chem. 8, 209-219 (2016).

9. Yu, X., Zhao, H., Xi, S., Chen, Z., Wang, X., Wang, L., Lin, L. Q. H., Loh, K. P. \& Koh, M. J. Site-selective alkene borylation enabled by synergistic hydrometallation and borometallation. Nat. Catal. 3, 585-592 (2020).

10. Camp, A. M., Kita, M. R., Blackburn, P. H., Dodge, H. M., Chen, C.-H. \& Miller, A. J. M. Selecting double bond positions with a single cation-responsive iridium olefin isomerization catalyst. J. Am. Chem. Soc. 143, 2792-2800 (2021).

11. Scaringi, S. \& Mazet. C. Kinetically controlled stereoselective access to branched 1,3-dienes by Ru-catalyzed remote conjugative isomerization. ACS Catal. 11, 7970-7977 (2021).

12. Murai, M., Nishimura, K. \& Takai, K. Palladium-catalyzed double-bond migration of unsaturated hydrocarbons accelerated by tantalum chloride. Chem. Commun. 55, 2769-2772 (2019).

13. Wang, Y., Qin, C., Jia, X., Leng, X. \& Huang, Z. An agostic iridium pincer complex as a highly efficient and selective catalyst for monoisomerization of 1-alkenes to trans-2-alkenes. Angew. Chem., Int. Ed. 56, 1614-1618 (2017).

14. Lin, L., Romano, C. \& Mazet, C. Palladium-catalyzed long-range deconjugative isomerization of highly substituted $\alpha, \beta$-unsaturated carbonyl compounds. J. Am. Chem. Soc. 138, 10344-10350 (2016).

15. Perdriau, S., Chang, M.-C., Otten, E., Heeres, H. J. \& de Vries, J. G. Alkene isomerisation catalysed by a ruthenium PNN pincer complex. Chem. - Eur. J. 20, 15434-15442 (2014).

16. Grotjahn, D. B., Larsen, C. R., Gustafson, J. L., Nair, R. \& Sharma, A. Extensive isomerization of alkenes using a bifunctional catalyst: An alkene zipper. J. Am. Chem. Soc. 129, 9592-9593 (2007). 
17. Kim, D., Pillon, G., DiPrimio, D. J. \& Holland, P. L. Highly Z-selective double bond transposition in simple alkenes and allylarenes through a spin-accelerated allyl mechanism. J. Am. Chem. Soc. 143, 3070-3074 (2021).

18. Yu, X., Zhao, H., Li, P. \& Koh, M. J. Iron-catalyzed tunable and site-selective olefin transposition. J. Am. Chem. Soc. 142, 18223-18230 (2020).

19. Zhang, S., Bedi, D., Cheng, L., Unruh, D. K., Li, G. \& Findlater, M. Cobalt(II)-catalyzed stereoselective olefin isomerization: facile access to acyclic trisubstituted alkenes. J. Am. Chem. Soc. 142, 8910-8917 (2020).

20. Kapat, A., Sperger, T., Guven, S. \& Schoenebeck, F. E-Olefins through intramolecular radical relocation. Science 363, 391-396 (2019).

21. Meng, Q.-Y., Schirmer, T. E., Katou, K. \& König, B. Controllable isomerization of alkenes by dual visible-light-cobalt catalysis. Angew. Chem. Int. Ed. 58, 5723 -5728 (2019).

22. Liu, X., Zhang, W., Wang, Y., Zhang, Z.-X., Jiao, L. \& Liu, Q. Cobalt-catalyzed regioselective olefin isomerization under kinetic control. J. Am. Chem. Soc. 140, 6873-6882 (2018).

23. Crossley, S. W. M., Barabé, F. \& Shenvi, R. A. Simple, chemoselective, catalytic olefin isomerization. J. Am. Chem. Soc. 136, 16788-16791 (2014).

24. Chen, C., Dugan, T. R., Brennessel, W. W., Weix, D. J. \& Holland, P. L. Z-Selective alkene isomerization by highspin cobalt(II) complexes. J. Am. Chem. Soc. 136, 945-955 (2014).

25. Rigo, P., Bressan, M. \& Basato, M. Nickel(0) complexes with the hybrid bidentate ligand 1-(thioethyl)-2(diphenylphosphino)ethane. Synthesis and catalytic properties of the related nickel hydride derivative. Inorg. Chem. $18,860-863$ (1979).

26. Lochow, C. F. \& Miller, R. G. Nickel-promoted isomerizations of alkenes bearing polar functional groups. J. Org. Chem. 41, 3020-3022 (1976).

27. Tolman, C. A. Chemistry of tetrakis(triethylphosphite)nickel hydride, HNi[P(OEt)3]4+. IV. Mechanism of olefin isomerization. J. Am. Chem. Soc. 94, 2994-2999 (1972).

28. Kuntz, E. G., Godard, G., Basset, J. M. \& Vittori, O. M. Nickel(0)-TPPTS-cyanide complex in water. An efficient and flexible catalyst for the isomerisation of olefinic compounds at room temperature. Oil Gas Sci. Technol. 62, 781-785 (2007).

29. Frauenrath, H., Reim, S. \& Wiesner, A. Asymmetric double-bond isomerization of cyclic allyl acetals by using diop and chiraphos modified nickel complexes. Tetrahedron: Asymmetry. 9, 1103-1106 (1998).

30. Wille, A., Tomm, S. \& Frauenrath, H. A highly Z-selective isomerization (double-bond migration) procedure for allyl acetals and allyl ethers mediated by nickel complexes. Synthesis. 1998, 305-308 (1998). 
31. Lee, W.-C., Wang, C.-H., Lin, Y.-H, Shih, W.-C. \& Ong, T.-G. Tandem isomerization and C-H activation: Regioselective hydroheteroarylation of allylarenes. Org. Lett. 15, 5358-5361 (2013).

32. Dible, B. R., Sigman, M. S. \& Arif, A. M. Oxygen-induced ligand dehydrogenation of a planar bis- $\mu$-chloronickel(I) dimer featuring an NHC ligand. Inorg. Chem. 44, 3774-3776 (2005).

33. Shevick, S. L., Wilson, C. V., Kotesova, S., Kim, D., Holland, P. L. \& Shenvi, R. A. Catalytic hydrogen atom transfer to alkenes: a roadmap for metal hydrides and radicals. Chem. Sci. 11, 12401-12422 (2020).

34. Green, S. A., Crossley, S. W. M., Matos, J. L. M., Vásquez-Céspedes, S., Shevick, S. L. \& Shenvi, R. A. The high chemofidelity of metal-catalyzed hydrogen atom transfer. Acc. Chem. Res. 51, 2628-2640 (2018).

35. Shaikh, T. M., Weng, C.-M. \& Hong, E. F. Secondary phosphine oxides: Versatile ligands in transition metalcatalyzed cross-coupling reactions. Coord. Chem. Rev. 256, 771-803 (2012).

36. Montchamp, J. L. Phosphinate chemistry in the 21st century: a viable alternative to the use of phosphorus trichloride in organophosphorus synthesis. Acc. Chem. Res. 47, 77-87 (2014).

37. Gallen, A., Riera, A., Verdaguer, X. \& Grabulosa, A. Coordination chemistry and catalysis with secondary phosphine oxides. Catal. Sci. Technol. 9, 5504-5561 (2019).

38. van Leeuwen, P. W. H. M., Cano, I. \& Freixa, Z. Secondary phosphine oxides: Bifunctional ligands in catalysis. ChemCatChem. 12, 3982-3994 (2020).

39. Ackermann, L. Air- and moisture-stable secondary phosphine oxides as preligands in catalysis. Synthesis. 10, 1557-1571 (2006).

40. Sutra, P. \& Igau, A. Anionic phosph(in)ito ("phosphoryl”) ligands: Non-classical "actor". Coord. Chem. Rev. 308, 97-116 (2016).

41. Martin, D., Moraleda, D., Achard, T., Giordano, L. \& Buono, G. Assessment of the electronic properties of $p$ ligands stemming from secondary phosphine oxides. Chem. Eur. J. 17, 12729-12740 (2011).

42. Dubrovina, N. V. \& Börner, A. Enantioselective catalysis with chiral phosphine oxide preligands. Angew. Chem. Int. Ed. 43, 5883-5886 (2004).

43. Han, L.-B., Zhang, C., Yazawa, H. \& Shimada, S. Efficient and selective nickel-catalyzed addition of H-P(O) and HS bonds to alkynes. J. Am. Chem. Soc. 126, 5080-5081 (2004).

44. Zhang, J.-S., Zhang, J.-Q., Chen, T. \& Han, L.-B. t-BuOK-mediated reductive addition of $P(O)-H$ compounds to terminal alkynes forming $\beta$-arylphosphine oxides. Org. Biomol. Chem. 15, 5462-5467 (2017).

45. Huang, Z., Liu, W., Li, S., Yang, Y., Guo, S. \& Cai, H. Potassium carbonate promoted nucleophilic addition of alkenes with phosphites. Synlett. 31, 1295-1297 (2020). 
46. Keglevich, G., Sipos, M., Takács, D. \& Greiner, I. A study on the Michael addition of dialkylphosphites to methylvinylketone. Heteroat. Chem. 18, 226-229 (2007).

47. Bunlaksananusorn, T. \& Knochel, P. t-BuOK-catalyzed addition phosphines to functionalized alkenes: a convenient synthesis of polyfunctional phosphine derivatives. Tetrahedron Lett. 43, 5817-5819 (2002).

48. Coudray, L. \& Montchamp, J.-C. Recent developments in the addition of phosphinylidene-containing compounds to unactivated unsaturated hydrocarbons: Phosphorus-carbon bond-formation via hydrophosphinylation and related processes. Eur. J. Org. Chem. 21, 3601-3613 (2008).

49. Mahamulkar, S. G., Cisarova, I. \& Jahn, U. The lithiation reactivity and selectivity of differentially branched alkyldiphenylphosphine oxides - a simple and versatile approach to ortho-functionalized arylphosphine oxides. Adv. Synth. Catal. 357, 793-799 (2015).

50. Gu, Y. \& Tian, S. Olefination reactions of phosphorus-stabilized carbon nucleophiles. Top. Curr. Chem. 327, 197238 (2012).

51. Clayden, J. \& Warren, S. Stereocontrol in organic synthesis using the diphenylphosphoryl group. Angew. Chem. Int. Ed. 35, 241-270 (1996).

52. Benaglia, M. \& Rossi, S. Chiral phosphine oxides in present-day organocatalysis. Org. Biomol. Chem. 8, 38243830 (2010).

53. Kotani, S., Sugiura, M. \& Nakajima, M. Enantioselective aldol reactions catalyzed by chiral phosphine oxides. Chem. Rec. 13, 362-370 (2013).

54. Ayad, T., Gernet, A., Pirat, J.-C. \& Virieux, D. Enantioselective reactions catalyzed by phosphine oxides. Tetrahedron 75, 4385-4418 (2019).

55. Bhattacharya, A. K. \& Rana, K. C. Design, synthesis and biological evaluation of peptidyl-vinylaminophosphonates as novel cysteine protease inhibitor. Bioorg. Med. Chem. 19, 7129-7135 (2011).

56. Finkbeiner, P., Hehn, J. P. \& Gnamm, C. Phosphine oxides from a medicinal chemist's perspective: physicochemical and in vitro parameters relevant for drug discovery. J. Med. Chem. 63, 7081-7107 (2020).

57. Leeuwen, P. W. N. M. v., Kamer, P. C. J., Claver, C., Pàmies, O. \& Diéguez, M. Phosphite-containing ligands for asymmetric catalysis. Chem. Rev. 111, 2077-2118 (2011).

58. Xie, C., Smaligo, A., Song, X.-R. \& Kwon, O. Phosphorus-based catalysis. ACS Cent. Sci. 7, 536-558 (2021).

59. Weix, D. J. Methods and Mechanisms for Cross-Electrophile Coupling of Csp2 Halides with Alkyl Electrophiles. Acc. Chem. Res. 48, 1767-1775 (2015). 
60. Keglevich, G., Henyecz, R. \& Mucsi, Z. Experimental and theoretical study on the "2,2-bipiridyl-Ni-catalyzed” Hirao reaction of $>\mathrm{P}(\mathrm{O}) \mathrm{H}$ reagents and halobenzenes: $\mathrm{A} \mathrm{Ni(0)} \rightarrow \mathrm{Ni}(\mathrm{II})$ or a $\mathrm{Ni}(\mathrm{II}) \rightarrow \mathrm{Ni}(\mathrm{IV})$ mechanism? J. Org. Chem. 85, 14486-14495 (2020).

61. Carreras, J., Caballero, A. \& Perez, P. J. Alkenyl boronates: Synthesis and applications. Chem. Asian J. 14, 329343 (2019).

62. Lim, D. S. W. \& Anderson, E. A. Synthesis of vinylsilanes. Synthesis 44, 983-1010 (2012).

63. Han, L.-B., Ono, Y. \& Yazawa, H. Nickel-catalyzed addition of $\mathrm{P}(\mathrm{O})-\mathrm{H}$ bonds to propargyl alcohols: One-pot generation of phosphinoyl 1,3-butadienes. Org. Lett. 7, 2909-2911 (2005).

64. Fischer, K., Jonas, K., Misbach, P., Stabba, R. \& Wilke, G. The "nickel effect". Angew. Chem. Int. Ed. 12, $943-953$ (1973).

65. Boulens, P., Pellier, E., Jeanneau, E., Reek, J. N. H., Olivier-Bourbigou, H. \& Breuil, P.-A. R. Self-assembled organometallic nickel complexes as catalysts for selective dimerization of ethylene into 1-butene. Organometallics 34, 1139-1142 (2015).

66. Sperline, R. P. \& Roundhill, D. M. New mixed metal chain complexes of platinum(II) with copper(II), cobalt(II), nickel(II), zinc(II), uranium(VI), and thorium(IV) having substituted phosphonito groups as bridging ligands. Inorg. Chem. 16, 2612-2617 (1977).

67. Roundhill, D. M., Sperline, R. P. \& Beaulieu, W. B. Metal complexes of substituted phosphinites and secondary phosphites. Coord. Chem. Rev. 26, 263-279 (1978).

68. Walther, B. The coordination chemistry of secondary phosphine chalcogenides and their conjugate bases. Coord. Chem. Rev. 60, 67-105 (1984).

69. Harvey, J. N. Electronic effects on the stability of isomeric alkyl transition metal compounds. Organometallics 20, 4887-4895 (2001).

70. Liu, C.-F., Wang, H., Martin, R. T., Zhao, H., Gutierrez, O. \& Koh, M. J. Olefin functionalization/isomerization enables stereoselective alkene synthesis. Nat. Catal. 4, 674-683 (2021).

\section{Acknowledgements}

This research was supported by the National University of Singapore Academic Research Fund Tier 1: R-143-000B57-114 (M.J.K.).

\section{Author contributions}

L.H. and E.Q.L. developed the catalytic method and carried out the mechanistic studies. M.J.K. directed the investigations and wrote the manuscript with revisions provided by the other authors. 
Page 16 